\title{
Un Ciclo de Mejora en el Aula de Didáctica de las Ciencias Experimentales: Investigando la Ciencia
}

\section{A Cycle of Improvement in the Experimental Sciences Didactics Classroom: Investigating Science}

GABRIELA DELORD

ORCID: https://orcid.org/0000-0003-2283-5976

Universidad de Sevilla

Departamento de Didáctica de la

Ciencias Experimentales y Sociales

gcattani1@us.es

DOI: http://dx.doi.org/10.12795/9788447231003.012

Pp.: 256-273 


\section{Descripción del Contexto}

Mi Ciclo de Mejora en el Aula (CIMA) fue impartido en la Facultad de Educación en el grado de Educación Primaria. El nombre de la asignatura es Didáctica de las Ciencias Experimentales y tiene como objetivo general enseñar a los futuros maestros que es la Ciencia y cómo se enseña, entre otros objetivos específicos. En total son 60 alumnos subdivididos en 3 subgrupos. Las clases empezaron de forma semipresenciales, pero el CIMA fue desarrollado de forma totalmente online, debido a las medidas de sanidad de Andalucía frente al COVID-19. El CIMA fue aplicado a los 60 alumnos, pero la evaluación del aprendizaje ha sido aplicada en uno de los subgrupos, que tenía 20 estudiantes.

En general, los alumnos no saben muy bien de que trata la asignatura, lo que se pretende que aprendan y lo que es la Ciencia y la relación de la asignatura con su futura carrera de maestro. Los estudiantes comentan, en el primer día de clase, mediante algunas preguntas que les hago, que no les gusta la ciencia pues tiene muchos nombres dificiles de memorizar y porque las clases en la escuela no eran dinámica ni interesantes. Así que, empiezo con un esfuerzo grande y con el compromiso docente de hacer que los alumnos cambien sus ideas previas sobre la ciencia y empiecen a interesarse por esta asignatura que en realidad tiene una gran relevancia social. Frente a este contexto, creo que el CIMA a cumplido su papel en enganchar a los estudiantes.

\section{Diseño del CIMA}

El CIMA fue diseñado a través de tres bloques principales. Cada bloque está representado por un problema de investigación, siendo cada problema el hilo conductor de la enseñanza. El CIMA está en coherencia con el aprendizaje basado en la investigación de conceptos, procedimientos y valores sobre la Ciencia (Delord, 2020).

Ciclos de Mejora en el Aula (2020). Experiencias de Innovación Docente de la US Esta obra se distribuye con la licencia Creative Commons 
Para desarrollar el mapa de contenidos incluimos tres problemas que han estructurado todo el aprendizaje. De cada problema, se resaltan los contenidos necesarios para contestarlos. Hay contenidos del tipo conceptual, procedimental y actitudinal, como podemos ver el la Figura 1.

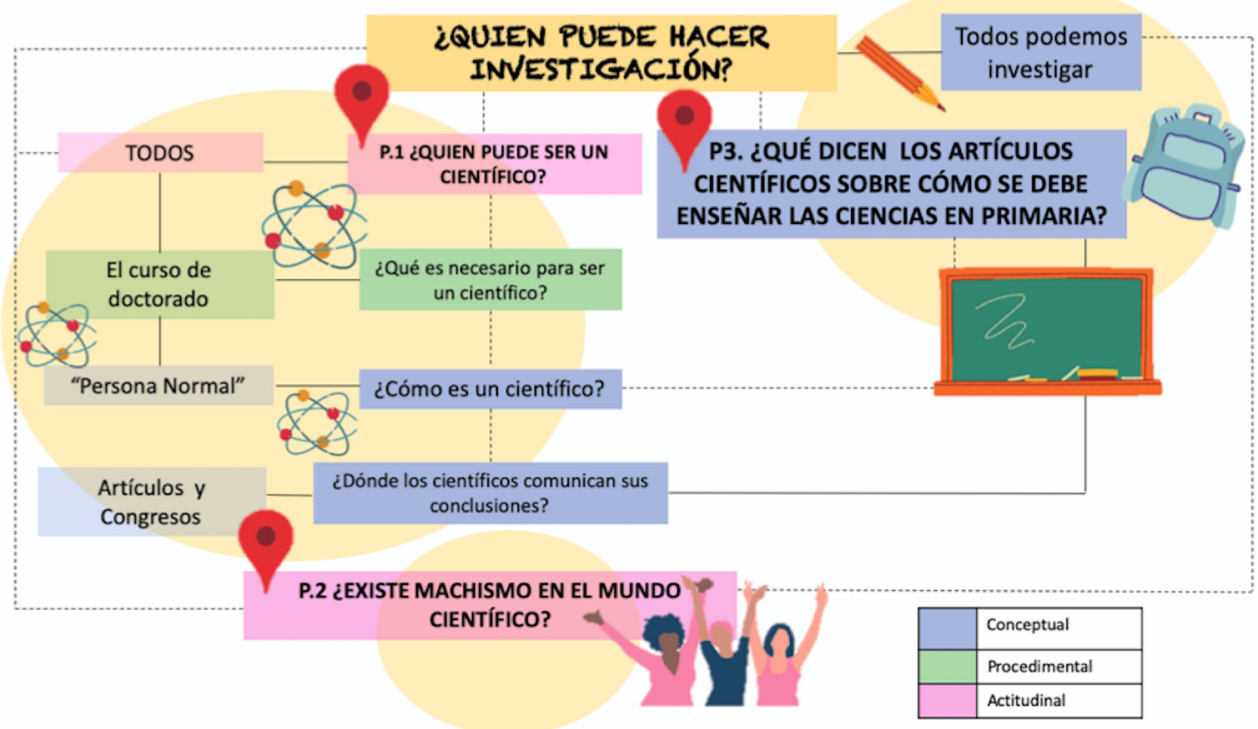

Figura 1. Mapa de contenidos y problemas

Modelo Metodológico Posible

Mí Modelo Metodológico Posible empieza con la "P" de "problema". Después de presentar el problema, los alumnos pasan al papel de protagonistas, ellos hacen la investigación de forma autónoma, desarrollando el "aprenden a aprender" (Bolívar, 2009). Por lo tato, pasamos a la fase de Investigación, "INV". Dentro de esta fase tenemos: Las hipótesis iniciales del alumnado en relación con el problema planteado, " $\mathrm{H}$ ", después hacemos la Actividad Contraste de Investigación, "ACI", que puede ser un video, búsqueda de

Ciclos de Mejora en el Aula (2020). Experiencias de Innovación Docente de la US Esta obra se distribuye con la licencia Creative Commons 
informaciones o un experimento para confirmar, rechazar o mejorar las hipótesis iniciales. El proceso termina con las conclusiones de lo que los estudiantes en pequeños equipos de trabajo han investigado. Así pasamos a la fase de "Con., que es de Conclusión y, después de esta, partimos a la comunicación de lo aprendido a los demás colegas, representado por "Com., de "Comunicación".

Para cerrar el proceso de enseñanza y aprendizaje, hago la sintesis con algunos comentarios necesarios. Después del cuestionario final, es posible identificar algún obstáculo o algunos alumnos que no han aprendido lo suficiente. Es en este momento que hago la etapa " $S$ ", de Sintesis. Abajo, Figura 2, el esquema de mi Modelo Metodológico.

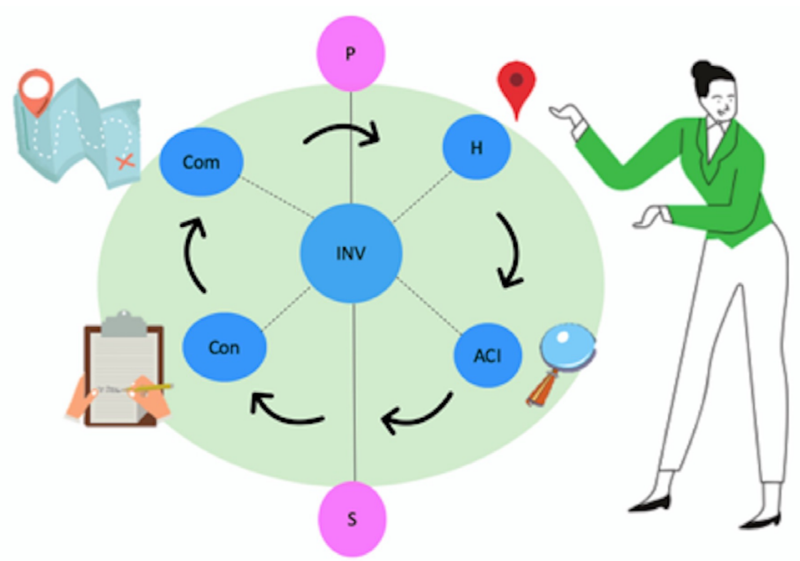

\begin{tabular}{|l|l|}
\hline $\mathbf{P}$ & Problema \\
\hline INV & Investigación, y dentro de esta investigación las siguientes etapas: \\
\hline H & Hipótesis de los alumnos \\
\hline ACI & Actividad de Contraste de Investigación \\
\hline Con. & Conclusión de la investigación \\
\hline Com. & Comunicación de lo investigado \\
\hline S & Síntesis del Aprendizaje \\
\hline
\end{tabular}

Figura 2. Modelo Metodológico Posible

Ciclos de Mejora en el Aula (2020). Experiencias de Innovación Docente de la US Esta obra se distribuye con la licencia Creative Commons 


\section{Secuencia de Actividades}

Como podemos percibir a través de las Tablas 1 y 2 , las secuencias de actividades son coherentes con el mapa de Contenidos y Problemas (Figura 1) y con el Modelo Metodológico Posible (Figura 2). Los alumnos están en el centro del aprendizaje (Carabaña, 2011) y son ellos los protagonistas.

Tabla 1. Secuencia de Actividades del Problema 1

\begin{tabular}{|c|c|c|}
\hline \multicolumn{3}{|c|}{ Problema 1: ¿Quien puede ser un científico? } \\
\hline & Actividades & Tiempo \\
\hline$P$ & $\begin{array}{l}\text { 1. Cuestionario Inicial. } \\
\text { 2. Presentación del Problema. }\end{array}$ & 30 min. \\
\hline $\mathrm{H}$ & $\begin{array}{l}\text { 3. Alumnos compartiendo sus hipótesis en pe- } \\
\text { queños equipos de trabajo y desarrollando en } \\
\text { equipo una hipótesis común } \\
\text { 4. Exposición de las hipótesis a los demás equipos. }\end{array}$ & $60 \mathrm{~min}$. \\
\hline $\mathrm{ACl}$ & 5. Los alumnos observan un vídeo de una científica. & $30 \mathrm{~min}$. \\
\hline Con. & 6. Realizan conclusiones. & $30 \mathrm{~min}$. \\
\hline Com. & 7. Exposición de las conclusiones. & 30 min. \\
\hline S & 8. Realización de una síntesis (docente). & $15 \mathrm{~min}$. \\
\hline
\end{tabular}

Tabla 2. Secuencia de Actividades de los Problemas 2 y 3

\begin{tabular}{|l|l|l|}
\hline \multicolumn{3}{|c|}{ Problema 2: ¿Existe machismo en el mundo científico? } \\
\hline $\mathrm{P}$ & \multicolumn{1}{|c|}{ Actividades } & Tiempo \\
\hline $\mathrm{H}$ & $\begin{array}{l}\text { 9. Presentación del Problema. } \\
\text { ñumnos compartiendo sus hipótesis en peque- } \\
\text { junto una hipótesis común. } \\
\text { 11. Exposición de las hipótesis a los demás equipos. }\end{array}$ & $10 \mathrm{min.}$ \\
\hline $\mathrm{ACl}$ & $\begin{array}{l}\text { 12. Los alumnos investigan la historia de vida de las } \\
\text { científicas más famosas (Google). Cada alumno } \\
\text { ha investigado una. }\end{array}$ & $30 \mathrm{~min}$. \\
\hline
\end{tabular}

Ciclos de Mejora en el Aula (2020). Experiencias de Innovación Docente de la US Esta obra se distribuye con la licencia Creative Commons Reconocimiento-NoComercial-SinObraDerivada Internacional (CC BY-NC-ND 4.0.) 


\begin{tabular}{|l|l|l|l|}
\hline Con. & $\begin{array}{l}\text { 13. Realizan conclusiones individuales sobre posi- } \\
\text { bles casos de machismo dentro de la carrera de } \\
\text { la científica investigada. }\end{array}$ & $30 \mathrm{~min}$. \\
\hline Com. & $\begin{array}{l}\text { 14. Exposición de las conclusiones a todos los } \\
\text { colegas. }\end{array}$ & $30 \mathrm{~min}$. \\
\hline $\mathrm{S}$ & $\begin{array}{l}\text { 15. Realización de una síntesis (docente) a través de } \\
\text { un vídeo-aula, que fue grabado y colgado la pla- } \\
\text { taforma YouTube. }\end{array}$ & $45 \mathrm{~min}$. \\
\hline Problema 3: ¿Qué dicen los artículos científicos de cómo se debe enseñar \\
\hline las ciencias en primaria?
\end{tabular}

Cuestionario Inicial y Final: Conociendo las ideas de los alumnos

La primera actividad, está centrada en la aplicación de un cuestionario inicial de forma anónima a los estudiantes (observar en la Tabla 1, Actividad 1). En este cuestionario tenemos las preguntas claves de cada problema del Mapa de Contenidos y Problemas. En total, han contestado 58 alumnos, y de estos, 20 han sido analizados, que representan el total de alumnos de un subgrupo. Por curiosidad, he realizado la lectura de las demás respuestas de los

Ciclos de Mejora en el Aula (2020). Experiencias de Innovación Docente de la US Esta obra se distribuye con la licencia Creative Commons 
otros cuestionarios y hay modelos de respuestas muy parecidos entre los tres subgrupos de estudiantes. En la Figura 3 tenemos el cuestionario aplicado al alumnado con un total de siete preguntas.

Tabla 3. Preguntas del cuestionario inicial y final

\begin{tabular}{|l|}
\hline 1. ¿Quién puede ser un científico? \\
\hline 2. ¿Qué es necesario para ser un científico? \\
\hline 3. ¿Cuáles son las características personales de un científico? \\
\hline 4. ¿Cómo se comunican los científicos? \\
\hline 5. ¿Solo los científicos hacen investigación? \\
\hline 6. ¿Crees que hay machismo en el mundo de las Ciencias? \\
\hline 7. ¿Qué dicen los científicos sobre cómo enseñar las Ciencias en Primaria? \\
\hline
\end{tabular}

\section{Aplicación del CIMA}

En general considero que el CIMA ha tenido una buena aceptación por parte de los estudiantes. El Mapa de Contenidos y Problemas me ha ayudado mucho a diseñar la secuencia de actividades. Sin embargo, más adelante, a través de las Escaleras de Aprendizaje, podemos percibir que una pregunta del cuestionario no estaba en el Mapa y por supuesto, no ha sido abordada en la secuencia de actividades, así que se nota el poco avance por parte de los estudiantes. Con esto podemos ver cuanto los docentes también nos equivocamos exigiendo algo de los alumnos, incluso culpabilizándolos, que no fue enseñado o que no fue bien enseñado, como en mi caso, con la pregunta 3 de cuestionario.

Quitando eso, el diseño del CIMA me ha dado mucha seguridad, la planificación del aula con todo diseñado y revisado por los formadores, me ha dado mucha seguridad en aplicarlo. No obstante, el diseño del CIMA ha sido

Ciclos de Mejora en el Aula (2020). Experiencias de Innovación Docente de la US Esta obra se distribuye con la licencia Creative Commons 
planificado para clases presenciales, y debido al COVID-19, las clases pasaron a ser todas online. En el diseño de las secuencias de actividades y a través de mi Modelo Metodológico, el alumnado ha sido el protagonista y las clases dependían mucho de su participación. En las clases presenciales es más fácil que participen, pero de forma online, con las cámaras y micrófonos apagados, no sabia si iban a participar y si las clases, el CIMA, podría convertirse en un desastre.

Felizmente, fue todo lo contrario, han participando de forma muy satisfactoria. A través de un diario fui apuntado el desarrollo de cada sesión. Los puntos positivos que he registrado en este diario fueron: a) Extrema puntualidad, antes de los 5 minutos que siempre espero para empezar de forma oficial la clase online, todos los alumnos ya estaban conectados; b) Participación muy activa de la mayoría; c) Motivación y enganche con las tareas; d) Tareas hechas para las sesiones y mucha responsabilidad y autonomía.

De la parte negativa o en relación con algún obstáculo, creo que es importante, cuando solicitamos tareas y explicamos estas de forma online y sabemos que solo vamos a verlos dentro de una semana, escribir correos con instrucciones. He visto que durante la semana surgían dudas y ellos me preguntaban casi todas las semanas. Pensando en la parte positiva de eso, se notaba el interés por hacer la tarea, que, además, no fue evaluada, no tenía puntos. Por el lado negativo, veo que yo podría haber solucionado estos problemas con correos informativos para dar a los alumnos más seguridad, pues ellos eran los protagonistas y creo que tomar este rol, que no es común para los estudiantes, debe ser tenido en cuenta por el docente, darles más seguridad con correos, guiones, pequeña explicación de la tarea en video, etc., Esto lo tendré en cuenta para mi próximo CIMA, dar más seguridad al alumnado.

Tengo algunas capturas de pantallas que demuestran la aprobación por el método aplicado. En una de ellas, pregunto a los alumnos directamente si estaban aprobando el 
trabajo. Todos contestarán que sí. En otra captura, una alumna avisa que no puede estar conectada, pero solicita la tarea del día. Por fin, una alumna hace la tarea y la envía por correo, pero no puede asistir a la clase y se preocupa de enseñar que ha hecho la tarea y expone que tiene una duda, lo que demuestra el interés en lo que estábamos estudiando. Como he mencionado anteriormente, me ha sorprendido la responsabilidad del alumnado frente a las tareas que no tenían ninguna puntuación. Es muy bonito y satisfactorio ver las ganas de aprender sin necesitar de un punto, solo por porque "yo quiero aprender". Además, no hay lista de asistencia, la asistencia a las clases online es voluntaria.

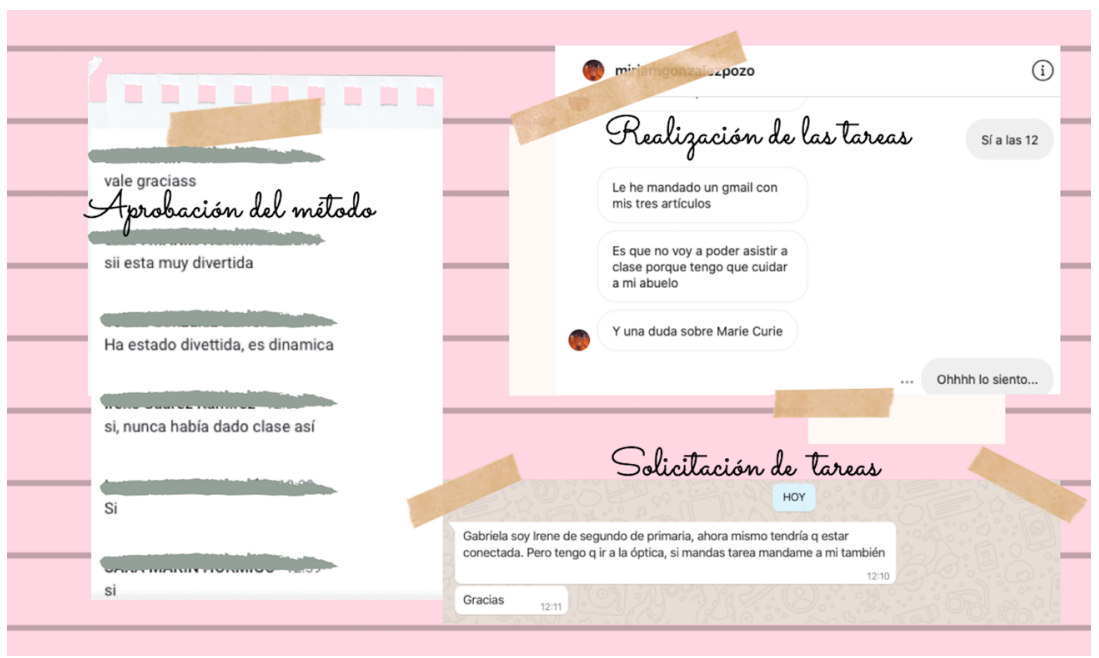

Figura 3. Opiniones de los alumnos sobre el método y algunas reacciones

Al final de cada clase online los alumnos, al despedirse, escribían en el chat Gracias Gabriela y otras frases similares de agradecimiento, no sé si los alumnos hacen eso con todos los docentes, pero, en general, en mis clases anteriores, los alumnos apenas decían adiós. He sentido estas frases como: gracias por enseñarme. Puedo estar equivocada, pero esta es la sensación que he tenido.

Ciclos de Mejora en el Aula (2020). Experiencias de Innovación Docente de la US Esta obra se distribuye con la licencia Creative Commons 
Pretendo seguir enseñando a través de mi nuevo modelo, presentado al principio de este capítulo, y pretendo al final pasar un cuestionario de evaluación de mi práctica docente, para tener seguridad, con datos mas rigurosos, de la aprobación o no por los estudiantes, a pesar que ya tengo algunas evidencias como he enseñado en la Figura 3.

\section{Sistema de Evaluación: Ideas de los alumnos antes y después}

Las actividades del CIMA no han sido calificadas con notas, pues han sido consideradas como actividades de aprendizaje. Las ideas finales de los estudiantes han sido utilizadas por mí cómo una sintesis general de todo lo aprendido y para "rescatar" a los alumnos que no han llegado a las ideas deseadas. Presento en lo que sigue algunas Escaleras de Aprendizaje y Evaluación de algunas de las preguntas del cuestionario inicial y final. También, los niveles de partida y llegada de cada alumno y los obstáculos identificados por mí. Con este método de evaluación he visto como los alumnos aprenden de forma satisfactoria, aunque no todos llegan al mismo nivel de aprendizaje, y me he propuesto "rescatar" a estos de forma democrática en la parte "S" de mí modelo metodológico, identificado como Síntesis.

Me gusta mucho la metáfora de que el docente es como se fuera un piloto de un avión, que debe llevar a todos al mismo destino con seguridad, sin saber quienes son, ni su sueños y objetivos, ni si tienen algún problema. Considero que las Escaleras de Aprendizaje cumplen con maestría este rol.

Para empezar, enseñaré la Escalera de la pregunta 1: ¿Quién puede ser un científico? Casi el 90\% de los alumnos, al principio, han contestado que para ser científico es necesario ser muy inteligente y que con eso es lo suficiente. A través del video de una científica ellos han visto que todas las personas pueden hacer la carrera de científico y 
que existen muchos tipos de programas de doctorado. Además, no es solo para las carreras de Química, Física y Biología, el abogado puede ser un científico de Derecho, por ejemplo. Siendo así, a través de este video, los alumnos han pasado del escalón 3 al escalón 4 en casi el 100\%, apenas un alumno ha permanecido en el escalón 3 y fue justo el alumno 5, que al principio estaba en el escalón 1, como podemos ver en la tabla de la izquierda. Por lo tanto, considero que la actividad del video de la científica ha sido exitosa.

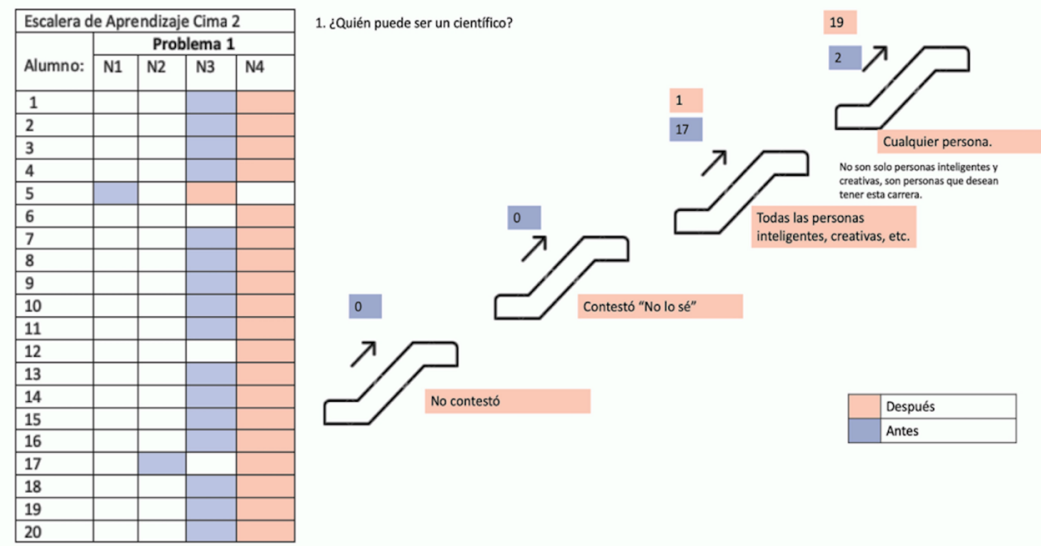

Figura 4. Escalera de Aprendizaje de la Pregunta 1

En la próxima escalera, presentaré la evolución de la pregunta del cuestionario numero 5: ¿La investigación es una actividad exclusiva de los científicos? La mayoría de los estudiantes al principio, han contestado que sí, en un total de 8 (ver escalón 2 o nivel 2 de la escalera, Figura 5), 5 estudiantes han dicho que estaban en duda (escalón o nivel 3 de la escalera, Figura 5) y 6 estudiantes ya sabían que todos podemos investigar, que no es algo exclusivo de los científicos (escalón o nivel 4, de la escalera, Figura 5). Después del video de la científica, tenemos un salto en el ultimo escalón. De 6 estudiantes, pasamos a tener 17 o sea, solo 3 estudiantes no llegaron al ultimo nivel de la Escalera.

Ciclos de Mejora en el Aula (2020). Experiencias de Innovación Docente de la US Esta obra se distribuye con la licencia Creative Commons 

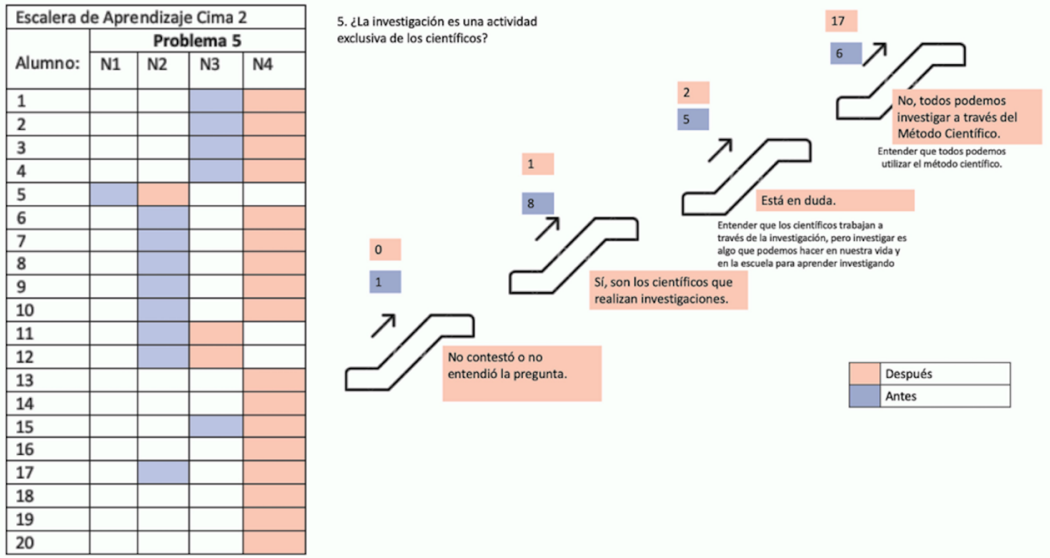

Figura 5. Escalera de Aprendizaje de la Pregunta 5

Ahora, les presento otra escalera en la que es muy evidente que los alumnos han evolucionado de una forma satisfactoria, lo que me permite enfatizar que es verdad que los alumnos pueden aprender por sí mismos, de forma autónoma e investigando. Las demás esclareas siguen en esta misma dinámica de evolución de las ideas de forma bastante homogénea. Por lo tano, no voy a presentarlas todas.

Sin embargo, hay una ultima escalera, después de esta, que me gustaría comentar, pues es la única en que los alumnos no han evolucionado de forma satisfactoria y pronto les contaré por qué. Pero antes, la penúltima escalera, comprobando una vez más que se aprende de forma alternativa a la tradicional. Después de la experiencia del CIMA estoy mucho más segura y convencida de que es posible enseñar y aprender investigando en el aula.

Ciclos de Mejora en el Aula (2020). Experiencias de Innovación Docente de la US Esta obra se distribuye con la licencia Creative Commons 

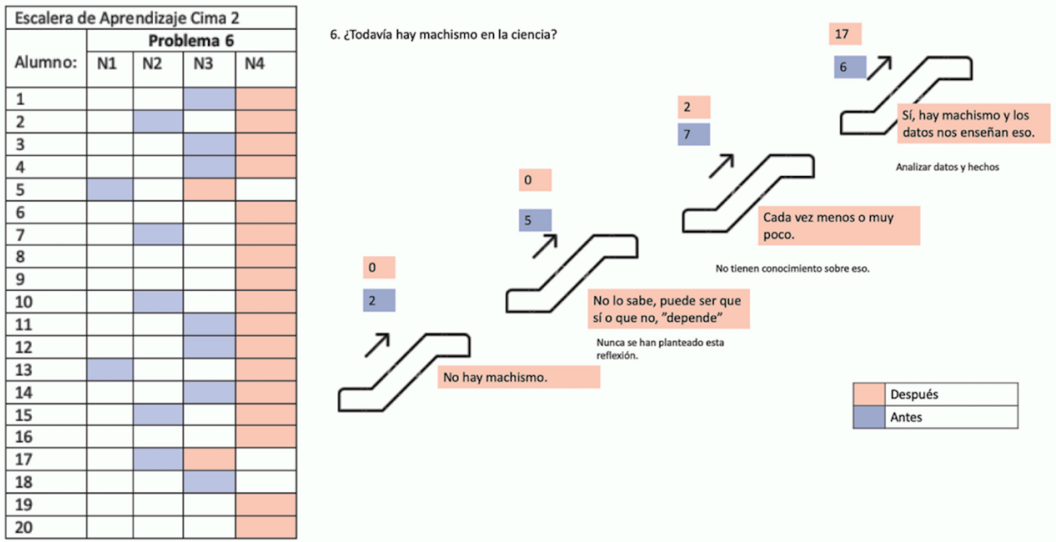

Figura 6. Escalera de Aprendizaje de la Pregunta 6

Como decía, presento la última escalera dónde podemos ver que la evolución de las ideas de los estudiantes no ha sido tan exitosa como en las demás. En esta ultima, que corresponde a la pregunta 3, ¿Cuáles son las características personales de un científico?, mi objetivo era enseñar que además de lo que la sociedad piensa, de que el científico debe ser alguien muy curioso, inteligente, etc., el científico debe ser antes de todo ético y honesto con los resultados de sus investigaciones. Lo que ha pasado es que no he realizado ninguna actividad especial, o con énfasis, en esta pregunta. En una parte del video de la científica ella comenta eso, pero de forma muy rápida. Así que podemos percibir que este conocimiento no ha sido desarrollado con éxito por mi parte. Al principio, cuando lo he visto, me quede un poco frustrada, pero parándome a analizar y mirar las secuencias de actividades, he descubierto que, a pesar de estar incluida en mi mapa, no he realizado ninguna actividad específica en relación con la pregunta. De esta forma, no sé pude pretender que los estudiantes avancen si no se les da la oportunidad adecuada para ello.

Ciclos de Mejora en el Aula (2020). Experiencias de Innovación Docente de la US Esta obra se distribuye con la licencia Creative Commons 


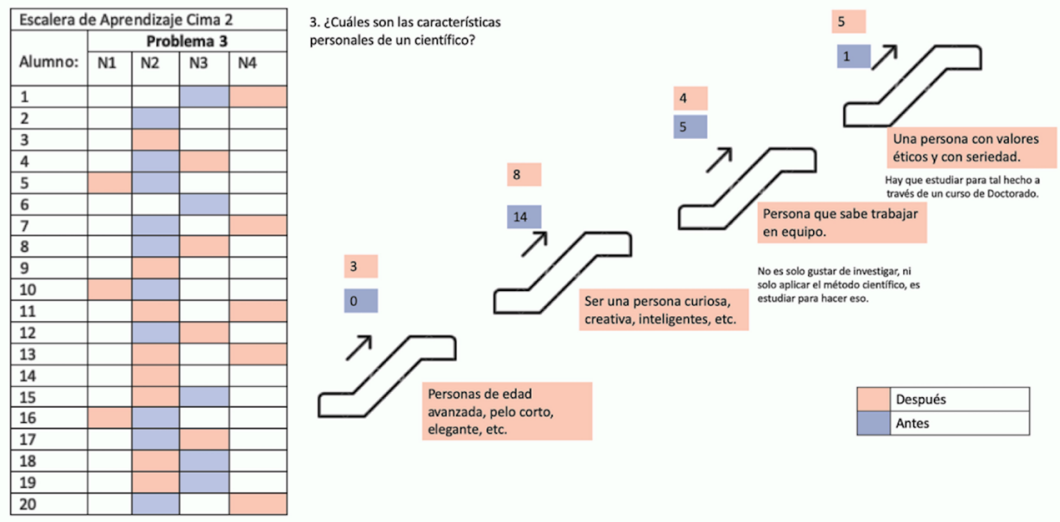

Figura 7. Escalera de Aprendizaje de la Pregunta 3

Por lo tanto, en esta escalera empezamos con 14 estudiantes diciendo que los científicos deben ser inteligentes, curiosos, etc., 5 han dicho que deben ser personas que deben trabajar en equipo, respuesta que jamás podría imaginar que la iban a decir, y una persona ha mencionado la seriedad, la ética y el respeto por la sociedad, respuesta que tan poco la esperaba. Al final, solo 5 alumnos de 20, han llegado al escalón 4 y 4 al escalón 3. Así que, de 20 sujetos, 9 han tenido un salto del escalón 2 para el 3, y 8 estudiantes se han quedado en el mismo escalón. Sin embargo, en escalón 1, el más bajo, al principio teníamos cero alumnos y ahora, al final aparecen 3 estudiantes. Eso realmente significa que esta temática no ha sido bien desarrollada.

\section{Evaluación del CIMA}

Conforme a lo que he comentado anteriormente, considero que el desarrollo del CIMA ha sido muy positivo. De hecho, pretendo seguir poniendo al alumnado en el centro del aprendizaje, seguir trabajando con la investigación, no solo en la enseñanza y aprendizaje sino también en mi propia práctica docente. Para eso, seguiré utilizando las Escaleras de Aprendizaje, que ha sido algo muy impactante,

Ciclos de Mejora en el Aula (2020). Experiencias de Innovación Docente de la US Esta obra se distribuye con la licencia Creative Commons 
además de los Mapas de Contenidos y Problemas, para ayudar en el desarrollo de mi Secuencia de Actividades. Además, pretendo mejorar en estos aspectos:

- Hacer correos electrónicos con explicaciones de las tareas. He llegado a la conclusión de que cuando se pretende que los alumnos sean los protagonistas, tenemos que apoyarlos y darles más seguridad.

- Desarrollar plantillas, tablas y guiones muy estructurados, para que los estudiantes realicen las tareas dentro de un marco homogéneo. Eso ayuda a que todos sepan lo que deben hacer y cómo hacerlo.

- Desarrollar pequeños ejemplos, para ayudar a entender lo que hay que hacer.

- Valorar más la importancia del trabajo cooperativo, pues a través de los pequeños equipos se enriquece la enseñanza y se multiplican los conocimientos.

\section{Todo lo que he aprendido}

- De los aspectos de la experiencia, lo que pretendo incorporar en mí practica docente habitual es: Mapa de Contenidos y Problemas. Muy importante para estructurar lo que realmente es necesario y lo que se pretende estudiar.

- Secuencia de Actividades coherentes con el Mapa de Contenidos y problemas.

- Cuestionario Inicial y Final coherente con la secuencia de actividades. He visto que no sé puede preguntar cosas que no se van a trabajar o a darles el énfasis adecuado. Así que repasaré el cuestionario teniendo como base el Mapa de Contenido y Problemas, confirmando que lo que se va a preguntar se va a enseñar.

- Además, seguiré utilizando las Esclareas de Aprendizaje en cada nuevo bloque de contenidos que empiece.

- Incorporaré más guiones informativos al alumnado, correos, plantillas y tablas para ayudar que las tareas de ellos sean más claras y uniformes, y con algunos 
ejemplos para que puedan trabajar de forma autónoma con seguridad.

- Por fin, llevaré más a menudo el diario de mis clases, para realizar ajustes y mejoras para los próximos años.

\section{Mis nuevos Principios Didácticos}

Algunos de principios ya los realizaba antes del CIMA, como, por ejemplo: enseñar a través de la investigación. Sin embargo, mi propia investigación docente no era realizada con tanto rigor. No utilizaba el diario de clase, solo a veces, no hacia un análisis sistemático de las ideas iniciales y finales del alumnado, pues apenas las corregía como correctas o equivocadas, y no hacia nada más que decir quien ha evolucionado y quien no, y dejaba a los que no habían evolucionado sin ninguna actividad de recuperación final. No me preocupaba "rescatar" a estos alumnos. Ahora, las escaleras me han servido para identificar no solo quien sabe, o cuantos saben más, sino para identificar también a los estudiantes que todavía no han llegado a los escalones más altos y para no pasar a un próximo bloque, sin darles una oportunidad más de nivelarse con sus demás colegas. Para mí, las escaleras me han despertado una sensación de enseñar con democracia, con justicia, sin prejuicios, y me he acordado mucho de las lecturas de Paulo Freire, al principio de mi carrera como docente.

Por lo tanto, mis nuevos principios didácticos son:

- Enseñar a través de la investigación (Carabaña, 2011). Los alumnos aprenden investigando (Escribano y Del Valle, 2008), se nota en las Escaleras de Aprendizaje que he realizado.

- Investigar mi práctica docente (Chocarro, Sobrino y Gonzáles-Torres, 2013; Giroux, 1990). Solo una investigación rigurosa puede ayudarnos a realizar cambios con

Ciclos de Mejora en el Aula (2020). Experiencias de Innovación Docente de la US Esta obra se distribuye con la licencia Creative Commons 
sentido. Por eso utilizaré el diario, la observación en el aula y las escaleras de aprendizaje.

- No realizar exámenes, no es democrático centrarse solo en los "buenos" alumnos, el aprendizaje debe ser para todos. Así que utilizaré las escaleras para ayudar a todos que no han conseguido subir de nivel, y no las utilizaré para destacar a los mejores y descalificar a los que no han llegado al nivel mas alto, al final esta es la mentalidad de la enseñanza tradicional (Delors, 1996) y no va de acuerdo con mis nuevos principios, de tener una enseñanza más democrática, para todos.

Palabras clave: Didáctica de las Ciencias Experimentales, Educación Primaria, Docencia Universitaria, Experimentación Docencia Universitaria, Ciencias.

Keywords: Didactics of Experimental Sciences, Primary Education, University Teaching, Experimentation of University Teaching, Sciences.

Ciclos de Mejora en el Aula (2020). Experiencias de Innovación Docente de la US Esta obra se distribuye con la licencia Creative Commons 


\section{Referencias Bibliográficas}

Bolivar, A. (2009). Aprender a aprender a lo largo de la vida. Multiárea. Revista de Didáctica, 4, 63-96.

Carabaña, J. (2011). Competencias y Universidad, o un desajuste por mutua ignorancia. Bordón, 63, 1, 15-31.

Chocarro de Luis, E.; Sobrino, A. y González-Torres, M.C. (2013). Scholarship of Teaching and Learning: un modelo de desarrollo profesional de los profesores universitarios. Revista Electrónica Interuniversitaria de Formación del Profesorado, 16(1), 5-14.

Delord, G.C. (202). Investigar en la Clase de Ciencias. Madrid: Morata

Delors, J. (1996). La Educación Encierra un Tesoro. Madrid: Santillana Ediciones.

Escribano, A. y Del Valle, A. (2008). El Aprendizaje Basado en Problemas. Una propuesta metodológica en Educación Superior. Madrid: Narcea.

Giroux, H. (1990). Los profesores como intelectuales: hacia una pedagogía crítica del aprendizaje. Barcelona: Paidós.

Unesco, (1998). Conferencia mundial sobre la educación superior: la educación superior en el siglo XXl. Visión y acción. (Tomo I, pp. 1-141). Disponible en: http://unesdoc.unesco.org/images/0011/001163/116345s.pdf.

Ciclos de Mejora en el Aula (2020). Experiencias de Innovación Docente de la US Esta obra se distribuye con la licencia Creative Commons 